\title{
Diabetes Tipo 2 e Estilo de Vida: o Papel do Exercício Físico na Atenção Primária e na Secundária
}

\author{
Type 2 Diabetes and Lifestyle: The role of exercise \\ for primary and secondary care
}

MARIANA SANTORo NAKagaki Discente do curso de especialização em Atuação Multiprofissional em Medicina do Exercício Físico e do Esporte, Departamento de Saúde Pública, Faculdade de Medicina, Universidade Estadual Paulista. E-mail: msantoro16@hotmail.com

Katia Cristina Portero MCLellan

Professora Assistente Doutor do Departamento de Saúde Pública, Faculdade de Medicina, Universidade Estadual Paulista/UNESP, Botucatu SP. E-mail: kmclellan@fmb.unesp.br
REsumo O diabetes mellitus (DM) é visto atualmente como epidemia mundial devido ao grande aumento em sua incidência e prevalência, cujo controle representa um grande desafio para os sistemas de saúde. Assim, ações de intervenção com foco na mudança do estilo de vida necessitam ser implementadas por esses sistemas. Considerando que o exercício e a dieta são os pilares do tratamento do DM, o objetivo desta revisão bibliográfica foi avaliar o papel da mudança do estilo de vida, mais especificamente do exercício físico, na atenção primária e secundária do DM tipo 2. Para tanto, embasou-se em artigos publicados na base de dados Scopus durante o período de 1991 a 2011. As palavras-chave pesquisadas, nas línguas portuguesa e inglesa, foram "diabetes mellitus tipo 2", "modificação no estilo de vida” e "papel do exercício físico". De acordo com os referidos artigos, a modificação no estilo de vida por meio da adoção de hábitos alimentares saudáveis, prática regular de exercícios físicos e controle de peso mostra-se altamente eficaz na prevenção, no controle e tratamento do DM tipo 2. Além disso, os exercícios físicos aeróbios, resistidos e combinados evidenciam melhoras no controle metabólico e consequente redução no risco de desenvolver DM tipo 2 e suas complicações.

Palavras-chave: DIABETES MELLITUS TIPO 2, MODIFICAÇÃO NO ESTILO DE VIDA, EXERCíCIO FÍsICO.

ABstraCT Diabetes Mellitus (DM) is currently seen as a worldwide epidemic due to the large increase in its incidence and prevalence, which control represents a major challenge for health systems. Thus, interventions focusing changes in lifestyle need to be implemented by those systems. Whereas exercise and diet are the cornerstones of treatment of diabetes, the aim of this review was to evaluate the role of change in lifestyle, specifically physical exercise, in primary and secondary attention of T2DM. For this purpose it was used in the Scopus database, considering the period from 1991 to 2011. The keywords searched in Portuguese and English were "type 2 diabetes mellitus", "change in lifestyle" and "role of physical exercise". In accordance with the above mentioned articles the change in lifestyle by adopting healthy eating habits, regular practice of physical activity and weight control was highly effective in the prevention, control and treatment of T2DM. Furthermore, the aerobic, resistance and combined exercises showed improvements in metabolic control and subsequent reduction in risk of developing T2DM and its complications.

Keywords: TYPE 2 DIABETES MELLITUS, CHANGE IN LIFESTYLE, PHYSICAL EXERCISE. 


\section{INTRODUÇÃO}

O diabetes mellitus (DM) é visto atualmente como epidemia mundial devido ao grande aumento em sua incidência e prevalência, cujo controle representa um grande desafio para os sistemas de saúde. Entre as causas dessa epidemia estão o envelhecimento da população, a urbanização crescente e a adoção de estilos de vida pouco saudáveis ${ }^{1}$.

Estima-se que a prevalência de DM entre adultos aumente substancialmente de 6,4\% (135 milhões de pessoas) em 2012 para $7,7 \%$ (439 milhões de pessoas) em 2030, e que tal aumento seja particularmente acentuado nos países em desenvolvimento ${ }^{2}$.

Todo este processo gera consequências humanas, sociais e econômicas arrasadoras, como 4 milhões de mortes por ano devido ao $\mathrm{DM}$ e às suas complicações, o que representa 9\% da mortalidade mundial total, aumento nos custos referentes ao tratamento da doença e, sobretudo, das complicações, além da redução na expectativa e qualidade e vida ${ }^{1}$.

Desse modo, no intuito de se prevenir o DM tipo 2, ações com enfoque na modificação do estilo de vida devem ser implementadas pelos serviços de saúde em nível nacional e internacional ${ }^{3}$.

A presença de condições crônicas, tais como: doença arterial coronária, doença arterial periférica, deficiência visual e depressão, em combinação com o diabetes, aumentam a incapacidade nos indivíduos ${ }^{4}$. Outros elementos incapacitantes comuns em diabéticos, como a redução da força e do tônus muscular e a intolerância ao exercício, devido à inatividade física e obesidade, constituem-se em importantes fatores modificáveis ${ }^{5}$.
Sabe-se que a adoção de hábitos de vida saudáveis, por exemplo, a prática regular de atividade física e um acompanhamento nutricional que enfatize o aumento de grãos, frutas, vegetais e a diminuição de gorduras saturadas na dieta são essenciais para a prevenção do $\mathrm{DM}$ e melhora na qualidade de vida $^{6}$, além de contribuírem para regulação da glicemia em pacientes com DM tipo $2^{7}$.

Diante do exposto, o objetivo desta revisão bibliográfica foi avaliar o papel da mudança do estilo de vida, mais especificamente do exercício físico, na atenção primária (prevenção) e na secundária (prevenção e controle das complicações) do DM tipo 2.

\section{Métodos}

Realizou-se uma revisão de artigos publicados na base de dados Scopus, considerando o período de 1991 a 2011. As palavras-chave pesquisadas, nas línguas portuguesa e inglesa, foram "diabetes mellitus tipo 2", "modificação no estilo de vida" e "papel do exercício físico"

Posteriormente, agrupou-se as informações em tópicos que abordam os seguintes assuntos: mudanças no estilo de vida, adesão à mudança no estilo de vida, a importância do exercício físico na prevenção, controle e tratamento do DM2, exercícios físicos aeróbios, exercícios físicos resistidos e exercícios físicos combinados.

\section{MUdANÇAS NO ESTILO DE VIDA}

O DM é uma doença crônica e as mudanças no estilo de vida ${ }^{8}$ são benéficas tanto para sua prevenção como para seu controle ${ }^{1}$.

O estilo de vida não saudável, composto por alimentação inadequada e inatividade fí- 
sica, constitui fator de risco comportamental e modificável ${ }^{1}$, sendo considerado uma das principais causas de morte evitáveis mundialmente ${ }^{9}$. Esse fator, associado à obesidade, que também é potencialmente modificável, eleva os riscos de desenvolvimento do DM tipo 2 e de suas complicações ${ }^{10}$.

Evidências mostram que $80 \%$ dos pacientes recém-diagnosticados diabéticos são obesos, para os quais o controle de peso torna-se ainda mais importante ${ }^{1}$, visto que a obesidade e o sobrepeso afetam a sensibilidade e secreção de insulina ${ }^{10}$.

Um estudo recente com 641 japoneses investigou a eficácia da modificação do estilo de vida na incidência do DM tipo 2 em indivíduos com concentrações plasmáticas de glicose de jejum alteradas. Após a redução da ingestão energética total, o aumento da atividade física e a diminuição de peso houve melhora na tolerância à glicose dos indivíduos avaliados. Além disso, a redução de peso, mesmo que modesta, foi benéfica o suficiente para a prevenção do DM tipo 2 em japoneses com sobrepeso ou obesidade moderada que apresentavam glicemia de jejum entre $100 \mathrm{a} 125 \mathrm{mg} / \mathrm{dl}^{3}$. Estudos de intervenção no estilo de vida em pessoas com tolerância diminuída à glicose mostraram uma redução entre $28,5 \%$ e $58 \%$ no risco de desenvolver diabetes ${ }^{11-13}$, sendo a mudança no estilo de vida (dieta e exercício físico) mais eficaz na prevenção primária do DM tipo 2 do que o uso de terapia medicamentosa ${ }^{13}$.

Nessa perspectiva, Pan et al. ${ }^{14}$ analisaram durante seis anos uma população de 577 homens e mulheres que apresentavam tolerância diminuída à glicose. O estudo mostrou redução do risco de desenvolver DM tipo 2 nos grupos que realizaram intervenção com dieta (31\%), com exercício físico $(46 \%)$ e dieta associada ao exercício físico (42\%). A intervenção no estilo de vida constituiu-se de adoção de hábitos alimentares saudáveis e prática regular de exercícios físicos.

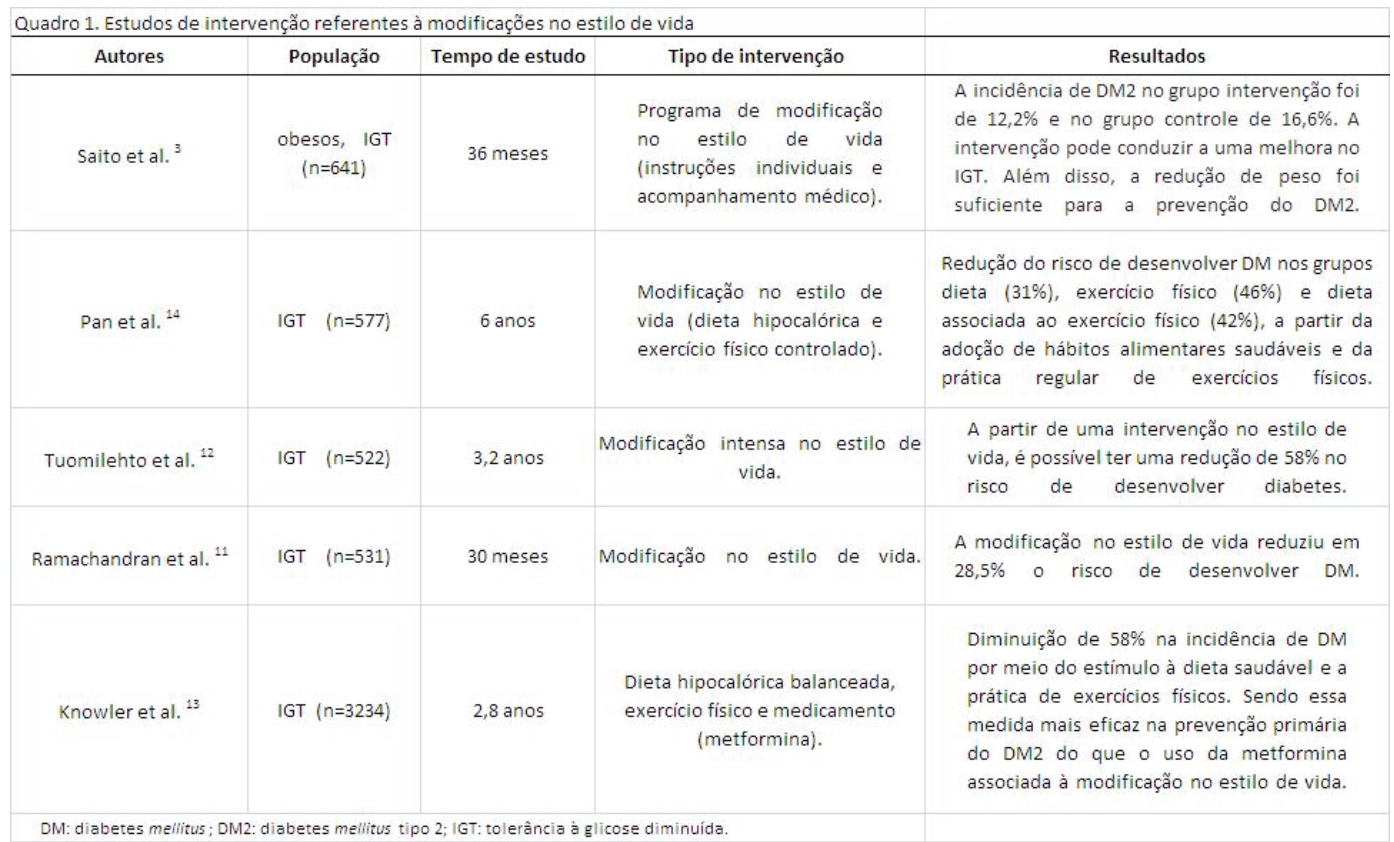


Nesse sentido, a educação alimentar desempenha um importante papel na prevenção primária e no tratamento do DM, tendo em vista que a alimentação saudável é essencial para o bom controle metabólico ${ }^{15}$ e pode contribuir para a redução dos valores de hemoglobina glicada entre $1-2 \%{ }^{1}$.

A hereditariedade, a obesidade, os hábitos alimentares, o estresse e a inatividade física apresentam-se como alguns dos fatores de risco modificáveis associados ao DM tipo 2. Tendo em vista que existem muitas evidências de que este possa ser prevenido por meio da mudança no estilo de vida ${ }^{8}$, o desafio dos sistemas de saúde consiste em desenvolver abordagens e estratégias eficazes no âmbito da saúde pública para proporcionar a incorporação dessa mudança na vida cotidiana ${ }^{16}$.

\section{Adesão À MUdAnÇA DO ESTILO DE VIDA}

O problema de adesão ao tratamento por parte dos indivíduos portadores de DM tipo 2 está relacionado tanto à falta de conhecimento acerca da doença quanto à inadequada capacitação dos profissionais da área de saúde envolvidos e à falta de integração entre eles ${ }^{17}$. Desse modo, a conscientização para a mudança de hábitos ${ }^{8}$, manutenção da autonomia e melhoria da expectativa e qualidade de vida por meio do acesso ao conhecimento tornam-se fundamentais durante o tratamento dessas pessoas ${ }^{15}$.

Vale ressaltar, ainda, que o sucesso de todo o tratamento está intimamente relacionado ao apoio da família e das pessoas mais próximas, as quais são essenciais para a efetivação das mudanças necessárias ${ }^{17}$.
Indivíduos com risco de desenvolver DM tipo 2 devem ser encorajados a participar de programas estruturados que enfatizam mudanças de estilo de vida (alimentação adequada e prática de exercícios físicos regulares). Essas alterações podem proporcionar perda de peso de $5 \%$ a $7 \%$ do peso inicial e reduzir o risco do desenvolvimento de DM tipo 2. De maneira geral, todos os membros da família desses indivíduos devem ser incentivados a realizar exercícios físicos regulares e ter uma alimentação adequada, com a finalidade de diminuírem o risco de desenvolver a doença ${ }^{10}$.

\section{IMPORTÂNCIA DO EXERCÍCIO FÍSICO NA ATENÇÃO PRIMÁRIA E NA SECUNDÁRIA DO DM TIPO 2}

Modificações simples no estilo de vida têm se mostrado altamente eficazes na prevenção ou no retardo do aparecimento do DM tipo 2. De acordo com Organização Mundial de Saúde ${ }^{18}$, as pessoas devem alcançar e manter peso corporal saudável, realizar dieta balanceada, evitar o uso de tabaco e ser fisicamente ativas.

Um estudo que investigou as relações entre atividade física, fatores fisiológicos e psicológicos em 109 mulheres negras com DM tipo 2 mostrou que mulheres inativas apresentaram maiores circunferências abdominais, índices de massa corporal (IMC) elevados, dores no corpo e menor vitalidade do que as mulheres ativas. Além disso, as mulheres que assistiam TV mais que duas horas por dia tinham concentrações plasmáticas significativamente mais altas de lipoproteína de alta densidade (LDL-c) e maior circunferência abdominal do que 
aquelas que assistiam TV menos do que duas horas por dia ${ }^{19}$.

Como já mencionado, a prática de exercícios físicos é fundamental para o tratamento do DM tipo 2 e pode reduzir muitos riscos a ele associados ${ }^{19}$. Realizar regularmente exercícios de intensidade moderada tem sido relacionado à diminuição no risco de doença coronária, hipertensão, acidente vascular cerebral, dislipidemia e resistência à insulina ${ }^{20}$. Esses benefícios são geralmente independentes do peso corporal ${ }^{20}$.

O tratamento da pessoa com DM tipo 2 deve envolver não apenas o médico, mas toda a equipe multiprofissional no intuito de contemplar fatores biológicos, emocionais, sociais e comportamentais que abrangem a vida do diabético ${ }^{1,8}$.

Existem evidências de que exercícios de intensidade moderada, realizados de três a cinco vezes na semana durante pelo menos 30 minutos, sejam benéficos para prevenir e controlar o DM tipo $2^{8}$. No entanto, o exercício físico não deve ser considerado separadamente de outros tratamentos, sendo que um resultado mais eficaz pode ser esperado apenas quando houver uma combinação ótima entre dieta, exercício físico e medicação ${ }^{21}$.

\section{Exercícios Físicos Aeróbios}

Exercícios aeróbios de intensidades baixa e moderada são os mais indicados no tratamento do DM tipo 2 pelo fato de atuarem positivamente na redução da glicemia, assim como na produção de insulina ${ }^{18}$.

Alguns outros estudos também evidenciaram os benefícios dos exercícios físicos no controle metabólico e estado de saúde de pessoas com DM tipo 2 (Quadro 2).

\begin{tabular}{|c|c|c|c|c|}
\hline Autores & População & Tempo de estudo & Tipo de intervenção & Resultados \\
\hline Ng et al. ${ }^{28}$ & $\begin{array}{l}\text { DM2, meia- } \\
\text { idade }(n=60) .\end{array}$ & 8 semanas & $\begin{array}{l}\text { TR X TA (de volume } \\
\text { e duração similares) }\end{array}$ & $\begin{array}{l}\text { Ambos os exercícios tiveram um impacto } \\
\text { positivo no estado de saúde geral dos } \\
\text { participantes da pesquisa. Parece que o TR } \\
\text { teve mais efeitos benéficos, como } \\
\text { mudanças significativas nos domínios do SF- } \\
36 \text { (questionário sobre o estado de saúde) } \\
\text { do que o observado no grupo de TA. }\end{array}$ \\
\hline Belli et al. ${ }^{24}$ & $\begin{array}{l}\text { Mulheres, } \\
\text { DM2, meia- } \\
\text { idade ( } n=19) \text {. }\end{array}$ & 12 semanas & $\begin{array}{c}\text { Caminhada no limiar } \\
\text { ventilatório }\end{array}$ & $\begin{array}{l}\text { Treinamento de caminhada em velocidade } \\
\text { de limiar ventilatório melhora em longo } \\
\text { prazo o controle glicêmico, a composição } \\
\text { corporal e capacidade de exercicio. }\end{array}$ \\
\hline Jorge et al. ${ }^{30}$ & DM2 $\quad(n=48)$. & 12 semanas & TCXTAXTR & $\begin{array}{l}\text { Melhora nos niveis de glicemia, perfil } \\
\text { lipidico, pressão arterial e hs-CRP. O TC e o } \\
\text { TR podem aumentar a expressão do IRS-1. }\end{array}$ \\
\hline Cuff et al. ${ }^{31}$ & $\begin{array}{l}\text { Obesas pós- } \\
\text { menopáusicas } \\
\text { com DM2 } \\
\quad(n=28) .\end{array}$ & 16 semanas & TC X TA & $\begin{array}{l}\text { TC melhorou a sensibilidade à insulina em } \\
\text { comparação ao grupo controle. TC e TA } \\
\text { reduziram PC e melhoraram VO2 máx. }\end{array}$ \\
\hline
\end{tabular}

DM2: diabetes mellitus tipo 2;TA: treinamento aeróbio ;TR: treinamento resistido;TC: treinamento combinado;hs-CRP: proteína C-reativa de alta sensibilidade; IRS-1: substrato receptor de insulina; PC: peso corporal. 
Araújo et al. ${ }^{22}$ investigaram o papel do exercício aeróbio no controle glicêmico em diabéticos. Os sujeitos foram submetidos à atividade aeróbia cinco vezes na semana durante uma hora cada dia. Foi observada redução significativa da glicemia após a intervenção do exercício abaixo do limiar anaeróbio. $\mathrm{O}$ estudo concluiu que o exercício aeróbio associado à alimentação saudável pode contribuir com o controle glicêmico de diabéticos.

No estudo de Vancea et al. ${ }^{23}$, os sujeitos foram divididos em três grupos, sendo eles: grupo controle (orientação e incentivo para a prática regular de exercício físico), grupo G3 (três sessões de exercício na semana) e grupo G5 (cinco sessões de exercício na semana), durante 20 semanas. O IMC e a porcentagem de gordura corporal (\%GC) dos grupos G3 e G5 reduziram significativamente durante o estudo, além de o G5 apresentar uma redução adicional na circunferência abdominal e glicemia. Desse modo, evidenciou-se um melhor resultado quando foi realizado o exercício físico moderado numa frequência de cinco vezes na semana para pacientes com DM tipo 2.

Um estudo com mulheres portadoras de DM tipo 2 investigou os efeitos de 12 semanas de um treinamento de caminhada dentro do limiar ventilatório sobre o controle metabólico. Resultados significativos foram observados acerca dos valores de $\mathrm{HbA1c}$, peso corporal, IMC, e VO2máx. O estudo sugere que o treinamento de caminhada em velocidade de limiar ventilatório melhora, em longo prazo, o controle glicêmico, a composição corporal e a aptidão física ${ }^{24}$.

Considerando-se a baixa adesão de pacientes diabéticos à prática regular de exercícios físicos, já é possível obter bons resultados por meio de exercícios aeróbios realizados três vezes na semana com intensidade moderada, preferencialmente durante 30 minutos $^{25}$. Colberg ${ }^{25}$ ainda adiciona que qualquer atividade física caracterizada como aeróbia pode colaborar com o controle glicêmico.

Vale ressaltar ainda que o fator motivacional é fundamental, visto que indivíduos envolvidos em atividades prazerosas podem se sentir mais motivados a continuar a sua prática. Nesse sentido, exercícios que envolvem grandes grupos musculares, como natação, ciclismo, dança, ou até mesmo exercícios como remar, patinar, pular corda e subir escadas, são alternativas que saem do convencional, ou seja, correr ou caminhar.

\section{EXERCícios Físicos RESISTIDOS}

Exercícios físicos resistidos têm se mostrado eficazes no controle glicêmico de pacientes com DM tipo $2^{26}$. Além disso, o treinamento resistido pode melhorar a função cardiovascular, diminuir fatores de risco associados à doença arterial coronariana, melhorar o bem-estar, promover a perda de peso e preservar a capacidade funcional ${ }^{27}$.

Um ensaio clínico randomizado comparou os efeitos do treinamento resistido progressivo (TRP) e do treinamento aeróbio (TA) em 60 indivíduos com DM tipo 2 com relação ao estado de saúde (mensurado por meio do questionário SF-36) deles durante oito semanas. O grupo de TRP realizou nove exercícios (três séries de 10 repetições) a $65 \%$ de $1 \mathrm{RM}$, enquanto que o grupo de TA foi submetido a 50 minutos de exercício 
aeróbio a 65\% de sua FCmáx. prevista para a idade. Ambos os exercícios tiveram impacto positivo no estado de saúde e melhora clínica do DM tipo 2, no entanto o grupo de TRP apresentou maiores mudanças nos domínios do SF-36 do que o de TA ${ }^{28}$.

Um ponto importante a ser lembrado diz respeito à criatividade do profissional responsável pelo acompanhamento do indivíduo com DM tipo 2. Em virtude da natureza da doença, torna-se essencial a criação e manutenção da motivação por meio de programas de exercícios prazerosos. Assim, alternativas como exercícios resistidos dentro de piscinas e diferentes tipos de lutas e ginásticas, com o intuito de sair da musculação convencional, são extremamente válidas.

\section{EXERCícios físIcos COMBINADOS}

Evidências mostram benefícios do treinamento combinado para o controle metabólico de indivíduos com DM tipo 2. Ensaios clínicos randomizados demonstram que o treinamento físico combinado possibilita a redução dos valores de $\mathrm{HbA} 1 \mathrm{c}$ mais do que o treinamento aeróbio ou resistido sozinhos ${ }^{29}$.

Um estudo comparou os efeitos de três diferentes modalidades de exercício (aeróbio, resistido e combinado) no controle metabólico, resistência à insulina, marcadores inflamatórios, adipocitocinas e expressão tecidual do substrato 1 do receptor de insulina (IRS -1) em 48 pacientes com DM tipo 2. Após 12 semanas de treinamento, os pacientes apresentaram diminuição da pressão arterial, da glicemia, do perfil lipídico e da proteína C-reativa ultrassensível
(PCR-us). Além disso, a expressão do IRS-1 aumentou em $65 \%$ no grupo resistido e em $90 \%$ no grupo combinado ${ }^{30}$.

O estudo de Cuff et al. ${ }^{31}$ avaliou durante 16 semanas os efeitos da combinação do treinamento resistido e aeróbio com relação à sensibilidade à insulina em 28 mulheres obesas pós-menopáusicas com DM tipo 2 . A combinação do treinamento resistido e aeróbio proporcionou melhoras significativas na sensibilidade à insulina, além de reduções de peso e obesidade abdominal em comparação ao grupo controle.

Os esportes como natação, basquete, vôlei, futebol, entre outros, são ótimos exemplos de exercícios combinados e, sobretudo, de alternativas que devem ser buscadas de acordo com a preferência de cada paciente, a fim de otimizar a adesão à prevenção e ao controle ou tratamento do DM tipo 2.

\section{Conclusão}

A adoção de hábitos de vida saudável com adequação alimentar e prática regular de exercícios físicos, independentemente do tipo, são fundamentais para a prevenção do DM tipo 2 e controle da glicemia. Evidências epidemiológicas e estudos de intervenção demonstram os benefícios de exercícios físicos aeróbios, resistidos, e combinados sobre o controle metabólico e estado de saúde de pessoas com DM tipo 2. Mesmo assim, a adesão à prática regular de exercício físico ainda é limitada; por isso, ações no âmbito da saúde pública devem ser estabelecidas para incentivar essa prática, contribuindo para a prevenção (atenção primária) e controle (atenção secundária) do DM tipo 2. 


\section{REFERÊNCIAS}

1. Ministério da Saúde. Diabetes Mellitus. Cadernos de Atenção Básica 2006; 16:56p.

2. Shaw JE, Sicree RA, Zimmet PZ. Global estimates of the prevalence of diabetes for 2010 and 2030. Diabetes Res Clin Pract 2010; 87:4-14.

3. Saito T, Watanabe M, Nishida J, Izumi T, Omura M, Takagi T, et al. Lifestyle modification and prevention of type 2 diabetes in overweight Japanese with impaired fasting glucose levels: a randomized controlled trial. Arch Intern Med 2011; 171:1352-60.

4. Park SW, Goodpaster BH, Strotmeyer ES, de Rekeneire N, Harris TB, Schwartz AV, et al. Decreased muscle strength and quality in older adults with type 2 diabetes: the health, aging, and body composition study. Diabetes 2006; 55:1813-8.

5. Larose J, Sigal RJ, Khandwala F, Kenny GP. Comparison of strength development with resistance training and combined exercise training in type 2 diabetes. Scand J Med Sci Sports 2011.

6. McLellan K, Lerário A, Burini R. Prevention of diabetes: effects of life style intervention. In: Zimering $\mathrm{M}$, editor. Topics in the prevention, treatment and complications of type 2 diabetes.; 2011. p. 163-89.

7. McLellan K, Barbalho S, Cattalini M, Lerario A. Diabetes mellitus do tipo 2, síndrome metabólica e modificação no estilo de vida. Revista de Nutrição 2007; 20:515-24.

8. Molena-Fernandes C, Nardo-Junior N, Tasca R, Pelloso S, Cuman R. A importância da associação de dieta e de atividade física na prevenção e controle do diabetes mellitus tipo 2. Acta Sci. Health Sci. 2005; 27:195-205.

9. Lopez AD, Mathers CD, Ezzati M, Jamison DT, Murray CJ. Global and regional burden of disease and risk factors, 2001: systematic analysis of population health data. Lancet 2006; 367:1747-57.

10. Costacou T, Mayer-Davis EJ. Nutrition and prevention of type 2 diabetes. Annu Rev Nutr 2003; 23:147-70.

11. Ramachandran A, Snehalatha C, Mary S, Mukesh B, Bhaskar AD, Vijay V. The Indian Diabetes Prevention Programme shows that lifestyle modification and metformin prevent type 2 diabetes in Asian Indian subjects with impaired glucose tolerance (IDPP-1). Diabetologia 2006; 49:289-97.

12. Tuomilehto J, Lindstrom J, Eriksson JG, Valle TT, Hamalainen H, Ilanne-Parikka P, et al. Prevention of type 2 diabetes mellitus by changes in lifestyle among subjects with impaired glucose tolerance. N Engl J Med 2001; 344:1343-50.

13. Knowler WC, Barrett-Connor E, Fowler SE, Hamman RF, Lachin JM, Walker EA, et al. Reduction in the incidence of type 2 diabetes with lifestyle intervention or metformin. N Engl J Med 2002; 346:393-403.

14. Pan XR, Li GW, Hu YH, Wang JX, Yang WY, An ZX, et al. Effects of diet and exercise in preventing NIDDM in people with impaired glucose tolerance. The Da Qing IGT and Diabetes Study. Diabetes Care 1997; 20:537-44.

15. Duran R, Soler Z, Santos B, Morraye M. Caracterização das Condições de Vida e Saúde dos Indivíduos Diabéticos Tipo II em uma Unidade de Saúde da Família. Investigação 2010; 10:S23- S30.

16. Walker KZ, O’Dea K, Gomez M, Girgis S, Colagiuri R. Diet and exercise in the prevention of diabetes. J Hum Nutr Diet 2010; 23:344-52.

17. Costa A, Balga R, Alfenas R, Cotta R. Promoção da saúde e diabetes: discutindo a adesão e a motivação de indivíduos diabéticos participantes de programas de saúde. Ciência e Saúde Coletiva $2011 ; 16: 2001-9$.

18. Organization $\mathrm{W}-\mathrm{WH}$. The scientific basis for diet, nutrition and the prevention of type 2 diabetes. Geneva, 2002:1-53. 
19. Allen NA, Melkus GD, Chyun DA. Physiological and behavioral factors related to physical activity in black women with type 2 diabetes mellitus. J Transcult Nurs 2011; 22:376-85.

20. Committee PAGA. Physical Activity Guidelines Advisory Committee Report. 2008.

21. Sato Y. Diabetes and life-styles: role of physical exercise for primary prevention. Br J Nutr 2000; 84 Suppl 2:S187-90.

22. Araújo R, Prada A, Córdova C, Prada F. O exercício físico no controle glicêmico de diabéticos frequentadores do programa pé-diabético no Hospital Regional de Taguatinga. Revista Brasileira de Ciência e Movimento 2009; 17.

23. Vancea DM, Vancea JN, Pires MI, Reis MA, Moura RB, Dib SA. Effect of frequency of physical exercise on glycemic control and body composition in type 2 diabetic patients. Arq Bras Cardiol 2009; 92:23-30.

24. Belli T, Ribeiro LF, Ackermann MA, Baldissera V, Gobatto CA, Galdino da Silva R. Effects of 12week overground walking training at ventilatory threshold velocity in type 2 diabetic women. Diabetes Res Clin Pract 2011; 93:337-43.

25. Coberg S. Atividade física e diabetes. São Paulo: Ed. Manole; 2002.

26. Hu FB, Sigal RJ, Rich-Edwards JW, Colditz GA, Solomon CG, Willett WC, et al. Walking compared with vigorous physical activity and risk of type 2 diabetes in women: a prospective study. Jama 1999; 282:1433-9.

27. Chodzko-Zajko WJ, Proctor DN, Fiatarone Singh MA, Minson CT, Nigg CR, Salem GJ, et al. American College of Sports Medicine position stand. Exercise and physical activity for older adults. Med Sci Sports Exerc 2009; 41:1510-30.

28. Ng CL, Tai ES, Goh SY, Wee HL. Health status of older adults with Type 2 diabetes mellitus after aerobic or resistance training: a randomised trial. Health Qual Life Outcomes 2011; 9:59.

29. Church TS, Blair SN, Cocreham S, Johannsen N, Johnson W, Kramer K, et al. Effects of aerobic and resistance training on hemoglobin A1c levels in patients with type 2 diabetes: a randomized controlled trial. Jama 2010; 304:2253-62.

30. Jorge ML, de Oliveira VN, Resende NM, Paraiso LF, Calixto A, Diniz AL, et al. The effects of aerobic, resistance, and combined exercise on metabolic control, inflammatory markers, adipocytokines, and muscle insulin signaling in patients with type 2 diabetes mellitus. Metabolism; 60:1244-52.

31. Cuff DJ, Meneilly GS, Martin A, Ignaszewski A, Tildesley HD, Frohlich JJ. Effective exercise modality to reduce insulin resistance in women with type 2 diabetes. Diabetes Care 2003; 26:2977-82.

Submetido em: 11/06/2012

Aceito em: 12/12/2012 